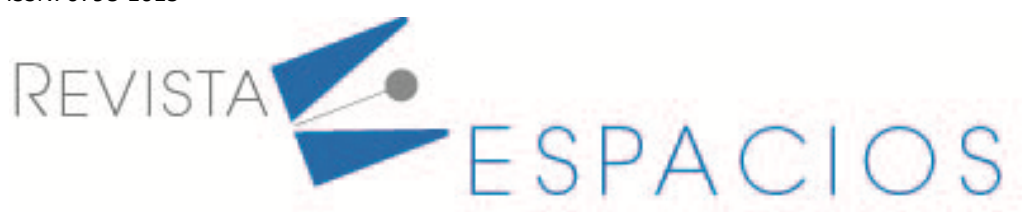

\title{
Maximização de lucros através da definição do mix ótimo de produção em uma panificadora: um estudo de caso em Marabá/Pa (Brasil)
}

\section{Profit maximization through the definition of the optimal production mix in a bakery: a case study in Marabá/Pa (Brazil)}

\author{
GOVEIA, Tamires G.S. ${ }^{1}$ \\ SANTOS, Izabela C.S. ${ }^{2}$ \\ SOUZA, Karina S. ${ }^{3}$ \\ SILVA, Jadielson G. F. ${ }^{4}$ \\ AQUINO, Danrley P. ${ }^{5}$ \\ TAVARES, Diego M. L. ${ }^{6}$
}

\section{Resumo}

A alta competitividade empresarial existente exige que as empresas se adaptem muito rapidamente ao mercado dinâmico, e tomem decisões rápidas diante dos mais diversos cenários econômicos. Para isso, fazer o uso das aplicações de pesquisa operacional se faz necessário para a tomada de decisões, uma vez que é possível através da matemática estabelecer critérios e obter resultados tangíveis, como por exemplo, maximizar lucro. A pesquisa em questão analisa os dados, sendo possível descrever de forma quantitativa os resultados obtidos.

Palavras-chave: programação linear, modelo matemático, panificadora, maximização de lucros.

\begin{abstract}
The existing high business competitiveness requires that companies adapt very quickly to the dynamic market, and make quick decisions in the most diverse economic scenarios. For this, making use of operational research applications is necessary for decision making, since it is possible through mathematics to establish criteria and obtain tangible results, such as maximizing profit. The research in question analyzes the data, and it is possible to describe the results obtained quantitatively.
\end{abstract}

Keywords: linear programming, mathematical model, bakery, profit maximization.

\footnotetext{
${ }^{1}$ Aluno de Graduação da Universidade do Estado do Pará. Brasil. tamires39.pbs@gmail.com

${ }^{2}$ Aluno de Graduação da Universidade do Estado do Pará. Brasil. slslaine.icss@gmail.com

${ }^{3}$ Aluno de Graduação da Universidade do Estado do Pará. Brasil. eukarinaps@gmail.com

${ }^{4}$ Aluno de Graduação da Universidade do Estado do Pará. Brasil. gabrielfarinha1999@gmail.com

${ }^{5}$ Aluno de Graduação da Universidade do Estado do Pará. Brasil. danrleypontes2@gmail.com

${ }^{6}$ Professor Mestre do curso de Graduação da Universidade do Estado do Pará. Brasil. diego.tavares@uepa.br
} 


\section{Introdução}

Diante da alta competitividade empresarial que avança perante o atual cenário econômico mundial, as empresas buscam por adaptações significativas, com isso surgiu a necessidade de medir a eficiência e eficácia dos seus processos produtivos para otimizá-los e garantir competitividade no mercado.

Para que uma organização mantenha a lucratividade, é necessário que haja um controle e planejamento de produção adequado, serviços e produtos com baixo custo e qualidade, além de estratégias que a possibilite alcançar as oportunidades que o seu nicho oferece, este desafio surge a partir de que existem " $n$ " entidades que atuam sob um mesmo seguimento.

A relação entre capital e trabalho é mediada pelo mercado, isto é, os lucros de uma empresa são obtidos através da diferença entre o custo de produção e o valor de venda do mercado, portanto para que a organização alcance seus lucros máximos, é necessário que toda a gestão seja elaborada a partir do princípio de maximização de lucros, uma vez que o mercado é instável e pode se alterar com facilidade.

Diante deste pensamento, existem diversos modelos capazes de auxiliar na definição de estratégias de um gestor, estes modelos de gestão possuem a missão de auxiliar os gestores a otimizarem seus resultados, possibilitando a alteração do mix de produção, objetivando o uso adequado de todos os recursos e capacidades instaladas (CASSEL et al., 2006).

Modelos de otimização matemática podem auxiliar à tomada de decisão estratégica, permitindo que os gestores sejam mais assertivos em seus controles e planejamentos, diante das mais variadas áreas que fazem o uso de modelos matemáticos, a pesquisa operacional (PO) é um estudo que além de desenvolver métodos analíticos, permite a aplicação prática nas diversas áreas de conhecimento (THE OPERATIONAL RESEACH, 2020).

Assim, o presente artigo tem a proposta de realizar a análise do processo produtivo de uma panificadora situada na cidade de Marabá/Pa que busca de forma contínua maneiras de diminuir seus custos, e aumentar seus lucros. Portanto, o objetivo desta pesquisa é de encontrar através de um modelo de programação matemática e estudos de pesquisa operacional, um mix ótimo de produção, isto é, encontrar uma sequência de produtos, quantidades e valores que garanta a maximização de lucros desta panificadora.

Para que os objetivos desta pesquisa fossem alcançados, ela foi estruturada da seguinte maneira: introdução, que descreve brevemente o tema abordado, assim como o problema e o objetivo do trabalho; referencial teórico, que aborda sobre os principais estudos necessários para a execução deste artigo, como: programação linear, pesquisa operacional entre outros; metodologia, onde está relatado de forma descritiva a realização do trabalho, desde a definição do tipo de estudo até a coleta de dados; os resultados, que apresenta os resultados obtidos mediante as analises executadas; e as considerações finais.

\section{Referencial Teórico}

\subsection{Pesquisa Operacional}

De acordo com Cardoso (2011) a Pesquisa Operacional (PO) é uma área do conhecimento onde se desenvolve métodos científicos com o objetivo de analisar, comparar e estabelecer estratégias ou tomadas decisões necessárias visando sempre bons resultados. A PO abrange inúmeras áreas, como a Estatística, a Economia, e a Informática, dessa forma, os especialistas que trabalham nessa área avaliam o problema em termos quantitativos obtendo assim uma solução precisa, ou seja, matematicamente ótima (DAVIS et al., 2001).

A aplicação da Pesquisa Operacional para a resolução de problemas dentro de uma organização consiste em basicamente definir o problema de maneira clara e precisa, determinar os fatores que acarretam esse problema 
e quantificar os fatores analisando cada um isoladamente, e é a partir disso que se obtém uma modelagem de operação para a resolução dos problemas encontrados (RODRIGUES et al., 2014). Assim, através desses processos matemáticos pode se ter a otimização dos processos, melhorando a eficiência organizacional.

\subsection{Programação Linear}

A Programação Linear (PL) é uma das técnicas mais utilizados para as resoluções dos modelos matemáticos de PO, ela é muito usada devido a sua simplicidade na formulação de modelos matemáticos, e resolução de problemas (GAMEIRO et al., 2011). Dessa maneira, ela permite estabelecer uma relação ótima de diversas variáveis segundo a uma função linear que um conjunto de restrições lineares para estas variáveis.

Assim, Corrar e Garcia (2001) mostram quais passos que devem ser seguidos para a criação do modelo matemático de Programação Linear:

modelo matemático de Programação Linear:

1. Determinar as variáveis de decisão;

2. Estabelecer o objetivo;

3. Determinar as relações básicas, especialmente restrições;

4. Calcular a solução ótima.

Para Colin (2007) o modelo é uma simulação da realidade de maneira simples que é descrita na forma de equações matemáticas. Assim, no modelo são encontradas: variáveis que servem para a tomada de decisão; função objetivo que relaciona as variáveis de decisão com o objetivo que deverá ser alcançado, tanto para maximizar (aumentar o lucro) ou minimizar (reduzir custos), logo, este resultado é chamado de solução ótima e as restrições são as limitações impostas para a realização do objetivo (BASTOS, 2019). Segundo Hillier e Lieberman (2013) o modelo deve apresentar: proporcionalidade, não negatividade, aditividade e separabilidade. Ou seja, a programação linear é um modelo matemático de otimização onde todas as funções são lineares, desta forma cada variável aparece na forma de K.X sendo K uma constante e X uma determinada variável. (CASTRO et al., 2015).

\subsection{Mix de Produção}

O mix de produção faz parte da programação linear pois é responsável por determinar o objetivo ótimo, como alcançar maior lucro ou maior receita de venda que se pode obter através da produção de determinado produto ou serviço, em situações que os recursos podem ser escassos ou limitados (LONGARAY, 2013 apud ANGELINI et al., 2017).

\subsection{Mix de Produção}

O Solver é um importante programa para análise hipotética de otimização onde podemos trabalhar com vários problemas de caráter linear e não linear, ele é uma das funções que possuem no Microsoft Excel assim como também nos softwares de otimização de programação linear Gurobi e Cplex. De acordo com Costa e Melo (2016), o programa resume a grupos de células as variáveis de decisão que satisfazem os limites das células de restrição e assim, por meio de interações matemáticas, produzem o resultado ótimo na célula da função objetiva. Ou seja, para a resolução de um problema no Solver é preciso defini-lo em forma de planilhas, logo em seguida especificar o valor da célula destino para que ela maximize ou minimize conforme a função objetivo, adicionar as variáveis e submeter as restrições, obtendo assim a solução ótima. 


\subsection{AIMMS}

O AIMMS (Advanced Integrated Multidimensional Modeling Software) surgiu em 1993, sendo uma ferramenta criada para contribuir com a modelagem matemática. Essa ferramenta auxilia as empresas em diversas áreas e também é usado nas universidades, pois, a mesma engloba as áreas de planejamento de produção, gestão, logísticas entre outras áreas. Segundo Baron (2017) o AIMMS é uma linguagem algébrica que é capaz de resolver problemas como programação quadrática, linear, não linear e inteira de alta complexidade. Dessa forma, o AIMMS é colocado como um dos mais relevantes pois está à frente de outras opções do mercado, pois é há uma facilidade com integração entre outras.

\section{Metodologia}

Essa pesquisa se fundamenta através da metodologia de estudo de caso, pois se caracteriza por uma pesquisa exploratória, sendo esta essencial para o estudo de temas que necessitam de um aprofundamento maior. Pois, como diz Ventura (2007) o estudo de caso é verificado nas pesquisas de teor exploratórias, e por ser um modelo que tem flexibilidade é a utilizado nas fases primárias dos estudos para a construção de hipóteses ou reformulação do problema.

O trabalho faz o uso de modelagem matemática para entender, calcular e analisar os dados coletados anteriormente através da análise exploratória, sendo assim um modelo quantitativo, uma vez que se trata de uma ferramenta para o auxílio de decisões racionais, estando ligado diretamente como objetivo desta pesquisa, que é maximizar os lucros a partir do encontro do mix ótimo de produção da organização.

As informações foram coletadas em uma panificadora que se situa na cidade de Marabá/PA, os dados coletados se trata de vendas e valores dos últimos 6 (seis) meses, destes compreende-se: agosto, setembro, outubro, novembro, dezembro de 2020 e janeiro de 2021. As análises dos dados foram feitas através de planilhas eletrônicas (Excel) e o software AIMMS para rodar e testar todos os dados, sendo estes coletados na observação in loco, em seguida foram calculadas as margens de contribuição de cada produto, definindo-se assim as restrições do sistema.

Para que o estudo fosse desenvolvido de forma eficaz, a metodologia se dividiu em algumas etapas, a fim de tornar a pesquisa mais confiável:

a) Coleta de dados: As coletas ocorreram através da contribuição intrínseca da empresa, uma vez que a mesma disponibilizou dados de vendas de produtos, quantidades vendidas, valores de custo, valores de venda, entre outros.

b) Escolha do problema e modelo de análise: O objetivo da organização supracitada se refere a maximizar os lucros, tendo como base esse propósito, se fez uma análise superficial de qual seguimento dentro da entidade mais contribuía para seus lucros mensais, e então definiu-se que seria feito um estudo para estabelecer o mix ótimo de produção no seguimento da empresa que mais lhe oferece rentabilidade, através dos estudos de pesquisa operacional.

c) Levantamento teórico: Foram feitas leituras de livros, artigos, publicações que pudessem contribuir com a base teórica, assim como avançar os conhecimentos que sustentem a abordagem do problema em questão, dando mais confiabilidade à pesquisa.

d) Aplicação do modelo: Execução dos softwares AIMMS e SOLVER para a análise dos dados, identificando nos programas as lacunas nas quais se faz a necessidade da implementação de programação linear para a descoberta do mix ótimo de produção. 
e) Análises de resultados: A partir dos dados coletados na aplicação dos modelos foi possível analisar e comparar os valores obtidos, verificando assim o método que mais se adere à execução do objetivo. Os dados utilizados nos softwares se referem ao: número de profissionais envolvidos, aos custos complementares, custos totais, entre outros.

O fluxo de execução desta pesquisa se contempla através de: coleta de dados, escolha do problema e modelo de análise, levantamento teórico, aplicação do modelo de solução, análises de resultados (Figura 1).

Figura 1

Fluxo de execução da pesquisa

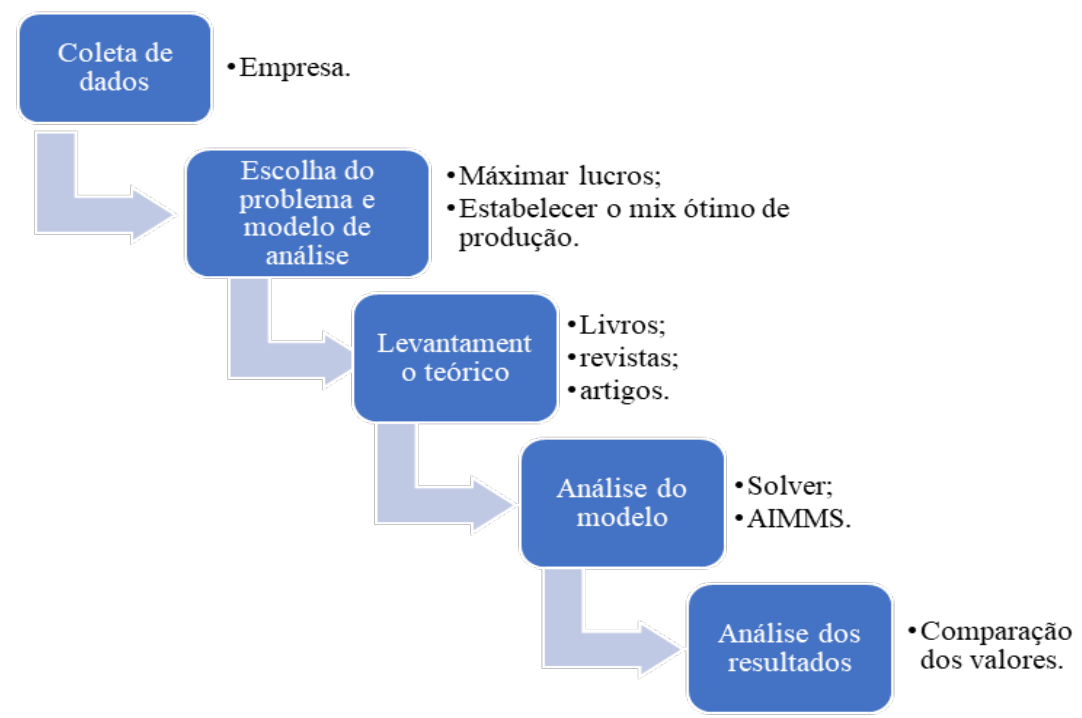

Fonte: Autores (2021)

\section{Descrição da Empresa}

O estudo foi realizado em uma panificadora localizada na cidade de Marabá- PA, onde é também uma conveniência que funciona 24 horas por dia, possuindo 40 (quarenta) funcionários em geral. A empresa em questão vende variados tipos de produtos e oferece diversos tipos de serviços, entre eles se destaca o buffet que dispõe de produtos como: salgado assado, salgado frito, bolo rocambole, torta doce, roscas e broas, pães ensacados e quitandas.

O buffet é vendido em quilogramas ( $\mathrm{kg}$ ) com preço único de $\mathrm{R} \$ 46,90$ (quarenta e seis reais e 90 centavos), no entanto, todos os produtos que fazem parte do buffet são terceirizados, assim sendo adquiridos de uma filial da mesma empresa, sendo comprados também em quilogramas, é importante destacar que os produtos são comprados a preço de custo.

Dessa forma, para atender a demanda relacionada a esse serviço a empresa trabalha com duas equipes de funcionários que intercalam os turnos de trabalho durante o dia, o total de pessoas responsáveis pelo buffet são de 31 (trinta e um) pessoas, compreendendo: 4 gerentes, 2 ajudantes de cozinha, 2 forneiros, 7 caixas e 17 atendentes. Cada equipe é composta por 1 gerente, 4 atendentes, 2 caixas, 1 forneiro e 1 ajudante de cozinha, que são responsáveis por preparar alguns produtos que chegam congelados da filial, e assim fazer a reposição dos mesmos durante o dia e atender os clientes. 


\section{Modelagem matemática}

A modelagem matemática está inclusa no que tange pesquisa operacional, uma vez que é através dela que se faz grande parte das análises necessárias de PO. Segundo Ignácio e Filho (2004) a PO se desenvolveu nos últimos anos diante de diversos modelos de algoritmos de otimização, todos eles buscando soluções de problemas em áreas como: a logística, produção, otimização etc.

Para satisfazer a resoluções de problemas é necessário o uso de modelos matemáticos, dado que estes são responsáveis pela elaboração de algoritmos, e segundo Matura et. al. (2004) estes modelos matemáticos são trabalhados de duas formas distintas, sendo que uma faz a utilização no nível estratégico, isto é, resolver problemas a longo prazo e mais complexos. E a outra maneira é a aplicação no nível tático/operacional, caracterizado por problemas que necessitam de um espaço de tempo mais curto.

Como o objetivo do artigo é de encontrar um mix ótimo de produção (com quantidades, valores etc.), a fim de maximizar os lucros da empresa supracitada, elaborou-se: conjuntos, parâmetros, variáveis, função objetivo (FO) e restrições para que se tornasse possível encontrar um resultado ótimo para a pesquisa. Foram criados 3 (três) sets, sendo eles: produtos, empregados e período. Assim como parâmetros para estabelecer as ligações entre os conjuntos. Foram também elaboradas variáveis para compor o problema (Vide Quadros 1, 2 e 3 respectivamente).

\section{Quadro 1}

Representação dos conjuntos

\begin{tabular}{|l|l|l|}
\hline Produtos & Empregados & Período \\
\hline Salgado assado & Gerente & Mês 1 \\
\hline Salgado frito & Atendente & Mês 2 \\
\hline Bolo de mesa/rocambole & Forneiro & Mês 3 \\
\hline Rosca/broa & Caixa & Mês 4 \\
\hline Pães ensacados & Ajudante de cozinha & Mês 5 \\
\hline Quitanda/sobremesas & & Mês 6 \\
\hline
\end{tabular}

Fonte: Os autores (2021)

\section{Quadro 2}

Representação dos parâmetros

\begin{tabular}{|c|c|}
\hline Parâmetro & Descrição \\
\hline Valkg & Valor por Kg \\
\hline $\operatorname{Cost} A q_{p}$ & Custo de aquisição \\
\hline NumEmp $p_{e}$ & número empregados \\
\hline ProdInd $_{p e}$ & Produção individual \\
\hline $\operatorname{CostEmp} p_{e}$ & salários \\
\hline TimeMonth $_{e}$ & Tempo mensal \\
\hline HourEx $_{e}$ & Horas Extras \\
\hline HourSub $_{e}$ & Horas Subcontratadas \\
\hline CapHourEx & Capacidade de horas extras \\
\hline CapHourSub & Capacidade e horas subcontratadas \\
\hline Costadm $_{e}$ & Custo de admissão \\
\hline
\end{tabular}




\begin{tabular}{|c|l|}
\hline Costfire $_{\boldsymbol{e}}$ & Custo de demissão \\
\hline Capacity $_{p}$ & Capacidade \\
\hline Dem & Demanda \\
\hline Valkg & Valor por Kg \\
\hline CostAq $_{p}$ & Custo de aquisição \\
\hline NumEmp & número empregados \\
\hline ProdInd & Produção individual \\
\hline CostEmp $_{e}$ & salários \\
\hline
\end{tabular}

Fonte: Os autores (2021)

Quadro 3

Representação dos variáveis

\begin{tabular}{|l|l|}
\hline Variáveis & Descrição \\
\hline$C_{e t}$ & Empregados em folha de pagamento no período t na função e; \\
\hline$H x_{e t}$ & Hora extra do empregado em folha de pagamento no período t na função e; \\
\hline$H s_{e t}$ & Hora subcontratada do empregado em folha de pagamento no período t na função e; \\
\hline$A_{e t}$ & Decisão de admitir um empregado no período t e na função e; \\
\hline$D_{e t}$ & Decisão de demitir um empregado no período t e na função e; \\
\hline$x_{t}$ & Decisão do quanto vender no período t; \\
\hline$y_{p e t}$ & Decisão do quanto comprar do produto e no período t; \\
\hline$C G_{e t}$ & Carga Horária. \\
\hline$C_{e t}$ & Empregados em folha de pagamento no período t na função e; \\
\hline$H x_{e t}$ & Hora extra do empregado em folha de pagamento no período t na função e; \\
\hline$H s_{e t}$ & Hora subcontratada do empregado em folha de pagamento no período t na função e; \\
\hline$A_{e t}$ & Decisão de admitir um empregado no período t e na função e; \\
\hline$D_{e t}$ & Decisão de demitir um empregado no período t e na função e; \\
\hline$x_{t}$ & Decisão do quanto vender no período t; \\
\hline$y_{p e t}$ & Decisão do quanto comprar do produto e no período t; \\
\hline
\end{tabular}

Fonte: Os autores (2021)

Em decorrência de todos os dados coletados acima fez-se a função objetivo (equação 1), logo em seguida as restrições que a acompanham.

$$
\begin{aligned}
& \operatorname{Max} \sum_{t \in T}\left(x_{t} \text { Valkg }\right)-\sum_{t \in T} \sum_{p \in P}\left(y_{\text {pet }} \operatorname{CostAq}_{p}\right)-\sum_{e \in E} \sum_{t \in T}\left(C_{e t} \operatorname{CostEmp}_{e}\right)- \\
& \sum_{e \in E} \sum_{t \in T}\left(\text { Hx }_{e t} \text { CostEmp }_{e}\right)-\sum_{e \in E} \sum_{t \in T}\left(H_{\text {et }} \operatorname{HourSub}_{e}\right)-\sum_{e \in E} \sum_{t \in T}\left(A_{\text {et }} \operatorname{Costadm}_{e}\right)- \\
& \sum_{e \in E} \sum_{t \in T}\left(D_{e t} \text { Costfire }_{e}\right)
\end{aligned}
$$




$$
\begin{aligned}
& \sum_{p \in P} \sum_{e \in E} y_{p e t}=x_{t} \\
& C_{e t} \geq \text { QuanMinFunc }_{e} \quad \forall e \in E, \mathrm{t} \in T \\
& \sum_{e \in E} Y_{p e t} \leq \operatorname{Dem}_{p t} \\
& C G_{e t}=\text { TimeMonth }_{e} * C_{e t}+H x_{e t}+H s_{e t} \\
& C_{e t}=C_{e t-1}+A_{e t}-D_{e t} \\
& C_{e 1}=\text { NumEmp }_{e}+A_{e, t}-D_{e, t} \\
& Y_{p e t} \leq \operatorname{ProdInd}_{p e} * C_{e t} \\
& Y_{\text {pet }} \leq \operatorname{ProdInd}_{p e} * C G_{e t} \\
& H x_{e t} \leq 0,20 * \text { TimeMonth }_{e} * C_{e t} \\
& H x_{e t} \leq \text { CapHourEx } \\
& H s_{e t} \leq \text { CapHourSub } \\
& H s_{e t} \leq 0,10 *\left(\text { TimeMonth }_{e} * C_{e t}+H x_{e t}\right) \\
& \sum_{t \in T} Y_{\text {pet }} \leq \text { Capacity }_{p} \\
& C_{e t}, H x_{e t}, H s_{e t}, A_{e t}, D_{e t} \geq 0 \\
& x_{t} \geq 0 \\
& y_{p t} \geq 0 \\
& \forall t \in T \\
& \forall p \in P, t \in T \\
& \forall t \in T, e \in E \\
& \forall t \in T, e \in E \mid \mathrm{t}>1 \\
& \forall t \in T, e \in E \\
& \forall p \in P, t \in T, e \in E \\
& \forall p \in P, t \in T, e \in E \\
& \forall t \in T, e \in E \\
& \forall t \in T, e \in E \\
& \forall t \in T, e \in E \\
& \forall t \in T, e \in E \\
& \forall p \in P, \mathrm{e} \in E \\
& \forall t \in T, e \in E \\
& \forall t \in T \\
& \forall t \in T, \mathrm{p} \in \mathrm{P}
\end{aligned}
$$

A função objetivo está sujeita a algumas restrições como: a Equação 2 se faz necessária, pois é através dela que se restringe a produção vendida, ou seja, tudo aquilo que é produzido deve ser vendido; a Inequação 3 restringe a quantidade mínima de empregados no período t; a Inequação 4 limita que aquilo que for adquirido no período t deve ser menor ou igual a demanda no período t; a Equação 5 define carga horária máxima de produção; a Equação 6 retrata o número de empregados na folha de pagamento, contabilizando as admissões e demissões em $t$ maior ou igual a 2 ( $\mathrm{t} \geq 2$ ); Inequação 7 determina a quantidade de colaboradores no período 1 ( $\mathrm{t}=1$ ) contabilizando o número de funcionários que já estão na empresa mais as admissões e demissões no mesmo período ; a Inequação 8 retrata sobre a produção individual de cada colaborador; a Restrição 9 restringe a compra de produtos como menor ou igual à relação entre a produção individual e a carga horária, isto é, só é possível comprar novos produtos se a empresa tiver capacidade para atender, caso contrário não é feita a aquisição; a Restrição 10 retrata a hora extra dentro dos limites estabelecidos por lei; a Inequação 11 delimita que as horas extras em $t$ devem ser menor ou igual a capacidade de hora extra estabelecida pela referida empresa; a equação 12 tem o mesmo princípio da Inequação 11, mas nesse caso se trata de horas subcontratadas; a Restrição 13 delimita as horas subcontratadas dentro do estabelecido pela empresa; a Inequação 14 relata que aquilo que for adquirido deve respeitar a capacidade do estabelecimento; as Restrições 15, 16 e 17 são de não negatividade. 
Tabela 2

Resultado da venda em $\mathrm{Kg}$

\begin{tabular}{|l|l|}
\hline Período & Kg. \\
\hline 1 & 3791.36 \\
\hline 2 & 2727.82 \\
\hline 3 & 3319.14 \\
\hline 4 & 4295.83 \\
\hline 5 & 4034.02 \\
\hline 6 & 4155.3 \\
\hline
\end{tabular}

Fonte: Autores (2021)

Todos os valores mostrados na Tabela 2 são referentes a venda dos produtos do buffet em cada período, podese notar que o período 4 é aquele em que mais se vende e que o período 2 o que é menos rentável para a empresa. Da mesma forma os resultados da variável de carga horária não ultrapassam os limites estabelecidos pelo modelo (Tabela 3), respeitando a carga horária total disponível para cada colaborador.

Tabela 3

Resultados

\begin{tabular}{|c|c|c|c|c|c|c|}
\hline Funções/período (mês) & $\mathbf{1}$ & $\mathbf{2}$ & $\mathbf{3}$ & $\mathbf{4}$ & $\mathbf{5}$ & $\mathbf{6}$ \\
\hline GERENTE & 2880 & 1440 & 1440 & 1440 & 1440 & 1440 \\
\hline CAIXA & 5760 & 1440 & 1440 & 1440 & 1440 & 1440 \\
\hline ATENDENTE & 12240 & 9360 & 9360 & 9360 & 9360 & 9360 \\
\hline FORNEIRO & 960 & 960 & 960 & 960 & 960 & 960 \\
\hline AJUDANTE DE COZINHA & 2160 & 720 & 720 & 720 & 720 & 720 \\
\hline
\end{tabular}

\section{Considerações Finais}

Os objetivos do artigo foram alcançados, pois foi possível encontrar um resultado para FO que compreendesse a maximização de lucros, respeitando todas as restrições estabelecidas, isto é, todos os resultados relatados acima chegam a um lucro de $\mathrm{R} \$ 376.085,26$. Ou seja, a escolha do modelo matemático foi satisfatória, pois atendeu as expectativas quantitativas da pesquisa.

Todos os dados utilizados nesta pesquisa são dados reais, e foram utilizados visando chegar o mais próximo da realidade, no entanto houve limitações no estudo, uma vez que os dados que foram obtidos diretamente dos próprios colaboradores da empresa, podendo haver falhas e alterações nos dados, sendo possível visualizar essa possibilidade através de uma análise mais profunda dos resultados. Devido ao cenário pandêmico que teve início no ano de 2019 e que perdura até o momento, não foi possível ir até o local e fazer a coleta dos dados pessoalmente.

A utilização do software AIMMS tornou o processo de análise do modelo mais simples, dado que o modelo possui muitas restrições e com o Solver de outros softwares não foi possível rodar com exatidão o modelo, sendo assim o AIMMS possibilitou de forma clara e suscita a análise e a apresentação dos dados obtidos.

Este estudo tem como serventia o embasamento para o auxílio de tomada de decisão que visão maximizar os lucros da empresa, visto que através dele foi possível demostrar a eficiência do uso de programação linear à tomada de decisão. 


\section{Referências bibliográficas}

Angelini J., Gechonke J., Ghazzawi B., Lourenço C., Soares T., Lima A. (2017). Utilização da Pesquisa Operacional para Otimização do Mix de Produtos na Produção de Picolés em uma Empresa de Foz do Iguaçu, PR. Pleiade, p. 90-98,

Baron C. (2017). UTILIZAÇÃO DE SOFTWARES NA PESQUISA OPERACIONAL. Revista Brasileira de Iniciação Científica. v.4(1), p. 162-170.

BASTOS M. M. (novembro, 2019). Aplicação da programação linear para otimização do mix de produção: estudo de caso de uma vendedora autônoma no rio de janeiro. Trabalho apresentado no XIX Simpósio De Pesquisa Operacional E Logística Da Marinha, Rio de Janeiro, Recuperado de https://www.marinha.mil.br/spolm/sites/www.marinha.mil.br.spolm/files

Cardoso, A. (2011). Fundamentos da Pesquisa Operacional. Belo Hotizonte Editora UNIFAL.

Davis, M. M., Aquilano, N. J., Chase, R. B (2001). Fundamentos de Administração da Produção. 3. ed. São Paulo: Bookman.

Corrar L. J., Garcia E. A. R. (novembro, 2001) Programação linear: uma aplicação à contabilidade de custos no processo de tomada de decisão. Trabalho apresentado no XXI Congresso Internacional De Custos, Fortaleza. Recuperado de https://www.intercostos.org/documentos/congreso-07/Trabajo066.pdf

Cassel R. A., Antunes J. A. V. (2006). Oenning, V. Maximização da lucratividade em produção conjunta: um caso na indústria frigorífica. Revista Produção, v. 16(2), p.244-257.

Castro, L.Y.; Borgert, A.; Souza, F.R. (dezembro, 2015). Definição do mix de produção em uma industria de lácteos com uso de programação linear: um estudo de caso. Trabalho apresentado no XXII Congresso Brasileiro de Custos, Foz do Iguaçu.

Colin, E. C. (2007). Pesquisa operacional: 170 aplicações em estratégia, finanças, logística, produção, marketing e vendas. Rio de Janeiro: LTC.

Costa L., Melo C. (novembro, 2016). Otimização de Treliças Metálicas Utilizando a Ferramenta Solver do Microsoft Excel. Trabalho apresentado no XXXVIII berian Latin-American Congress on Computational Methodsin Engineering, Brasília.

Gameiro A. H., Rocco C. D., Caixetafilho J. V. (julho, 2011). Modelo matemático para otimização e avaliação de unidade produtora de leite caracterizada pela integração lavoura-pecuária: consideração de parâmetros econômicos, logísticos e ambientais. Trabalho apresentado no Il Congresso Da Sociedade Brasileira De Administração E Sociologia Rural, Belo Horizonte.

Hillier, F. S., Lieberman G. (2013). Introdução à pesquisa operacional. 9. ed. Porto Alegre: AMGH.

Ignácio A., Filho V. (2004). O uso de software de modelagem aimms na solução de problemas de programação matemática. Pesquisa Operacional. v.24, p. 197-210.

Matura A., Ferrer J. C., Barañao F. (2004). Design and implementation-based decision support system generator. European Journal of Operational Research, v.154, p.170-183.

Rodrigues L., Ahlert F., Lacerda D. P., Camargo L. F. R., Lima P. N. (2014). Pesquisa Operacional - programação linear passo a passo: do entendimento do problema à interpretação da solução: do entendimento do problema à interpretação da solução. Brasil: Unisinos. 
The Operational Reseach. What is Operational Research?, 11 set. 2020. Disponível em: <https://www.theorsociety.com/about-or/>. Acesso em: 27 jan. 2021.

Ventura M. (2007). O Estudo de Caso como Modalidade de Pesquisa. Pedagogia Médica. p.383-386.

Esta obra está bajo una Licencia Creative Commons

Attribución-NoCommercial 4.0 International

(cc) EY-NC 